\title{
Contents, Vol. 104, 1942
}

\section{Index}

Anastassoff, A., Sur les inflammations oculaires d'origine

focale 136

Babel, Jean, Contribution à $\Gamma$ etude histologique du rhu-

matisme oculaire 243

Bakker, A., Ueber den Verlauf gesonderter Pupillarfasern

im Sehnerv 233

Bangerter, A., Ein Instrument zur Erleichterung der

Schleimhautnaht bei Dacryocystorhinostomie . . . 171 - Erfahrungen mit dem «Lochfinder» bei der Ablatio-

Operation 213

Bürki, E., Die therapeutische Verwendung des Privins

(Ciba) in der Ophthalmologie 254

Garabédían et Meunier, P., L'adaptométrie. Méthode cli-

nique pour le dépistage des carences nutritives, les

avitaminoses A en particulier 65

Goedbloed, J'., Mode of Inheritance in Chorioideremia . . 308 Goldmann, H., und Hagen, R., Zur direkten Messung der

Totalbrechkraft des lebenden menschlichen Auges . 15 Hagen, R., vide Goldmann, H.

Halbertsma, K. T. A., Arteriosklerotische Opticusatrophie . 289 Kull, J., Die Anwendung von

Prostigmin in der Glaukom-

behandlung 23

Meunier, P., vide Garabédian.

Mihályhegiji, G., Bedeutung der Sehschärfevermindening

für die Erwerbsfähigkeit und die Berufswahl . . 185 Pinkhof, J., Infantile Nuclear Aplasia

(Moebius) . . 177 Poleff, L., Zur Frage der Reaktion von Weil-Felix bei

Trachom . 121

Radnót, M., Der Augenbefund bei Cushingscher Krankheit 301 Saubermann, G. B. C, Ueber die Unterschiedsempfindlich-

keit des dunkeladaptierten Auges 157

-- vide Schmíd, A. E.

Schmid, A. E.i und Saubermann, G. B. C, Einwirkung desPermeabilitätsfaktors (Faktor P) auf den Augendruckbei normalen und hydrophthalmischen Kaninchen-augen 201

Weekers\} L., et Weekers, R., Mode d'action des operationsantiglaucomateuses, spécialement de la diathermisationdu corps ciliaire 1

Weekers, R., Le Glaucome incomplet. Contribution à Гétude

du Glaucome sans hypertension 316

Weinstein, P., Production of Aqueous Humor and Development of Glaucoma 166

Wínkler-Prins, C, Postoperativer Astigmatismus. Seine 
Entstehung und Vorbeugung 86

UEBERSICHTSREFERATE - REVIEWS - REVUES

Bruckner, A., Physikalische Optik. Dioptrik des Auges. Refraktion. Akkommodation. Methoden zur Refrak-tionsbestimmung. Brillenkunde. (Berichtszeit 1937 bis

1940) $217,264,332$

Coppez, L., Nystagmus (1938) 102

van Veelen, Pathologische Anatomie. (Berichtsjahr 1937) 32

GESELLSCHAFTSBERICHTE - SOCIETY TRANSACTIONS - SOCIÉTÉS

Sitzungsbericht der 103. Versammlung der Niederländischen Ophthal-mologischen Gesellschaft in Utrecht am 13. und 14. Dezember1941. Von C. 0. Roelofs, Amsterdam 48

NECROLOGIA

E.v. Emil v. Grósz $† 175$

Buchbesprechungen - Books Review - Livres Nouveaux 62

176

230

288 